\title{
Damage spreading in two-dimensional trivalent cellular structures with competing Glauber and Kawasaki dynamics
}

\author{
Z. Z. Guo ${ }^{1,2}$ and Kwok Yip Szeto ${ }^{1, *}$ \\ ${ }^{1}$ Department of Physics, Hong Kong University of Science and Technology, Clear Water Bay, Hong Kong, China \\ ${ }^{2}$ Department of Physics, Inner Mongolia Normal University, Hohhot 010022, China
}

(Received 14 December 2004; published 16 June 2005)

\begin{abstract}
The damage spreading of the Ising model on several two-dimensional trivalent structures, including soap froth, Voronoi, and hierarchical structures, are studied with competing Glauber and Kawasaki dynamics. The damage spreading transition temperature $T_{\mathrm{d}}$ and the Curie temperature $T_{C}$ of these structures are compared. We find that $T_{\mathrm{d}}$ of the hierarchical lattices decreases sharply as the probability of occurrence of Kawasaki dynamics increases, whereas for soap froth and Voronoi, $T_{\mathrm{d}}$ for the Voronoi and soap froth remain nearly unchanged except when the dynamics is dominated by Kawasaki dynamics. $T_{\mathrm{d}}$ and $T_{C}$ in our two-dimensional structures are nearly the same and they behave similarly as we change the relative probability of occurrence of the Glauber and Kawasaki dynamics. A heuristic argument is provided to explain the numerical results.
\end{abstract}

DOI: 10.1103/PhysRevE.71.066115

PACS number(s): $05.50 .+\mathrm{q}, 75.10 . \mathrm{Hk}, 75.40 . \mathrm{Mg}, 75.10 . \mathrm{Nr}$

\section{INTRODUCTION}

The damage spreading (DS) technique has been extensively applied in the study of dynamical properties of statistical models [1-5]. The possibility of applying the DS technique to simulate many economic and social phenomena has also been considered $[5,6]$. Generally, DS process in a given pattern can be simulated using various models in statistical mechanics; here we address the DS for the Ising model. Our aim is to obtain some insights on the relation between topologies and dynamics, in the context of damage spreading of the Ising model.

Trivalent structures have been investigated for many years due to their topological stability and general occurrence in nature. Recently, we have generalized the works of DS on regular lattices, such as the square [1,2], triangle [3], and cubic [4] lattice, to two-dimensional trivalent cellular patterns [5], for which soap froth is a typical model system that occurs in nature. Soap froth and Voronoi $[7,8]$ tile the plane with irregular polygons which have different areas. In order to have controllable topologies, we have also generalized the artificial trivalent structures constructed by polygons with different areas using the star-triangle transformation, resulting in a series of hierarchical regular lattices. Our work follows the examples of the 4-8 [9,10] and the 4-6 [11] lattice, which tile the plane with regular polygons of two kinds. The set of hierarchical regular lattices we obtained by startriangle transformation on hexagonal lattice results in a series of hierarchical lattice: the 3-12, the 3-6-24, and the 3-612-48 lattices. This kind of lattice has some interesting features in terms of the relation between topologies and the damage spreading using Glauber dynamics [5]. However, in the investigation on damage spreading for the economic and social systems, we find that using the Glauber dynamics has some limitations, as in many realistic cases, injection of energy by external force cannot be ignored. Since Kawasaki

\footnotetext{
*Corresponding author. Email address: phszeto@ust.hk
}

dynamics can be used to describe the effect of external source of energy, it is natural to extend our studies on DS by considering a combination of the Glauber and Kawasaki dynamics. Indeed, many recent works have addressed this issue of competing Glauber and Kawasaki (GK) dynamics for the Ising systems $[12,13]$. In this paper, we will focus our attention on the DS on various two-dimensional trivalent structures with competing GK dynamics. We find that the topology plays an important role in determining the DS transition temperatures, and there is characteristic difference between the hierarchical structures and the more natural and random cellular structures such as soap froth.

Many authors had discussed the relation between the DS transition temperature $T_{\mathrm{d}}$ and the Curie temperature $T_{C}$. This relation is of great importance. Till now, many methods have been suggested for the accurate determination of the DS transition temperature. A direct measure of the temperature dependence function of the averaged damage $\langle D\rangle$ can be used, but not with high accuracy $[3,5]$. On the other hand, one may use the peak location of the fluctuation $\sigma_{D}(T)$ of damage to define $T_{\mathrm{d}}$ [14]. More reliable estimate for the transition temperature can be obtained by using the finite-size scaling procedure $[14,15]$. All these methods will become obsolete if we can find the relation between $T_{\mathrm{d}}$ and $T_{C}$. For the twodimensional Ising model, it is shown that the heat-bath dynamics $T_{\mathrm{d}}$ coincides with $T_{C}$ whereas for Glauber and Metropolis dynamics $T_{\mathrm{d}}$ is near but smaller than $T_{C}[16,17]$. Unfortunately, satisfactory explanation for this relation between these two temperatures is still lacking. Thus, it is natu$\mathrm{ral}$, and of general interest, to consider if there is any relation between $T_{\mathrm{d}}$ and $T_{C}$ under competing Glauber and Kawasaki dynamics. This forms the second objective of our present investigation. In Sec. II, we present the general theoretical framework for our DS discussion. In Sec. III, the DS results are described. The effects of triangles on DS are studied in Sec. IV, and the relation between $T_{\mathrm{d}}$ and $T_{C}$ is discussed in Sec. V. 


\section{THEORY}

For a given two-dimensional trivalent cellular pattern, we put an Ising spin at the center of each cell of the pattern and perform Monte Carlo simulation using the Hamiltonian of nearest-neighbor Ising model

$$
H=-\sum_{\langle i, j\rangle} J_{i j} s_{i} s_{j}
$$

where $J_{i j}>0$ is the ferromagnetic exchange interaction coefficient between the nearest-neighbor sites $i$ and $j$, i.e., the interaction between the nearest-neighbor agents (polygons). In general, $J_{i j}$ is related with their areas [5]. In order to simplify our calculation we ignore the area influence, so that $J_{i j}=J$ for all of the nearest-neighbor sites.

We consider two identical systems A and B. First, we evolve system $\mathrm{A}$ for a long time to reach equilibrium, then system $\mathrm{B}$, which is a replica of system $\mathrm{A}$, is made. At $t=0$, the spin in the center cell of the lattice B is flipped (damaged) and fixed for all $t>0$. The Hamming distance (or damage) in phase space for the trivalent structures is calculated by

$$
D(t)=\frac{1}{M} \sum_{i=1}^{M}\left(1-\delta_{s_{i}^{A}(t), s_{i}^{B}(t)}\right)
$$

where $\left\{s_{i}^{A}(t)\right\}$ and $\left\{s_{i}^{B}(t)\right\}$ are the two spin configurations of the system which are subject to the same thermal noise and the same set of random numbers. Here $M$ is the number of the total spins on the lattice studied.

We use a combination of Glauber and Kawasaki dynamics (GK) to define the evolution of our systems. We first define a weighted transition probability per unit time from state $s$ to $s^{\prime}$ as

$$
\omega\left(s, s^{\prime}\right)=p \omega_{G}\left(s, s^{\prime}\right)+(1-p) \omega_{K}\left(s, s^{\prime}\right)
$$

The first term of the right side of Eq. (3) is based on Glauber dynamics, corresponding to a situation where the system is in thermal equilibrium with a heat reservoir. We go through the $M$ sites with a probability

$$
\omega_{G}\left(s, s^{\prime}\right)=\sum_{i=1}^{M} \delta_{s^{\prime}{ }_{1} s_{1}} \delta_{s^{\prime}{ }_{2} s_{2}} \cdots \delta_{s^{\prime}{ }_{i}-s_{i}} \cdots \delta_{s^{\prime}{ }_{M}{ }^{s} M} \omega_{i}(s)
$$

with $\omega_{i}(s)=\min \left\{1, \exp \left(-\Delta E_{i} / k_{B} T\right)\right\}$, where $\omega_{i}(s)$ is the probability of flipping spin $i$. The contact with the heat reservoir at temperature $T$ follows the Metropolis prescription, with $\Delta E_{i}$ being the change in energy in flipping spin $i$.

The second term of the right side of Eq. (3) is based on Kawasaki dynamics using two-spin exchange with the probability

$$
\omega_{K}\left(s, s^{\prime}\right)=\sum_{\langle i, j\rangle}^{M} \delta_{s^{\prime}{ }_{1} s_{1}} \cdots \delta_{s^{\prime}{ }_{i} s_{j}} \cdots \delta_{s^{\prime}{ }_{j} s_{i}} \cdots \delta_{s^{\prime}{ }_{M}{ }^{s_{M}}} \omega_{i j}(s),
$$

with

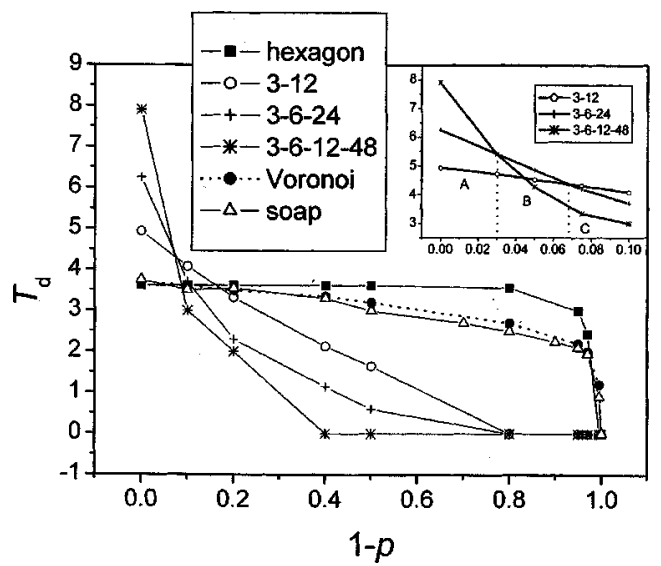

FIG. 1. The critical temperature of damage spreading with 1-p, in which $N=25,25,15$, and 15 for the hexagonal, the $3-12$, the 3-6-24, and the 3-6-12-48 lattice, respectively.

$$
\omega_{i j}(s)= \begin{cases}0 & \text { for } \Delta E_{i j} \leqslant 0, \\ 1 & \text { for } \Delta E_{i j}>0,\end{cases}
$$

where $\omega_{i j}(s)$ is the probability of exchange between the nearest-neighbor spins $i$ and $j$. If the change in energy $\Delta E_{i j}$ after exchanging the neighboring spins $i$ and $j$ is positive, the new configuration is automatically accepted. If it is negative, then the new configuration is not accepted. Kawasaki dynamics simulates a system subjected to a continuous flux of energy.

Also starting from Eq. (1), the Ising systems on the above structures may evolve through the paramagneticferromagnetic phase transitions under the competing dynamics. By calculating the Binder's cumulants with standard numerical procedure [18], we can obtain the Curie temperatures of these Ising systems. The Binder's cumulant is written as

$$
U_{N}=1-\frac{\left\langle m^{4}\right\rangle}{3\left\langle m^{2}\right\rangle^{2}}
$$

with $m=\left|(1 / N) \sum_{i} s_{i}\right|$, where $\langle\cdots\rangle$ represents the thermal average.

\section{DAMAGE SPREADING UNDER THE COMPETING DYNAMICS}

The results of the DS transition temperature $T_{\mathrm{d}}$ of the averaged damage spreading (the results are averaged over 100 configurations) of various trivalent structures as the function of 1- $p$ are depicted in Fig. 1. We have chosen the temperature when $\langle D\rangle$ rises to its half height of the value in the long time limit as the critical temperature (see the inset of Fig. 2); in this way, $T_{\mathrm{d}}$ for the soap structure with $p=1.0$ is approximately 3.75 ). Note that the DS transition temperature is dependent on the size of the structure. For soap and Voronoi structures, the size of the system is expressed by the ratio $F$, as sketched in Fig. 3. Here we have assumed the damage spreads from the center of the disk. For hierarchical structures, the size is expressed by $N$ (see Fig. 4). (Figures for the hierarchical lattices can be found in Ref. [5], where $N$ 


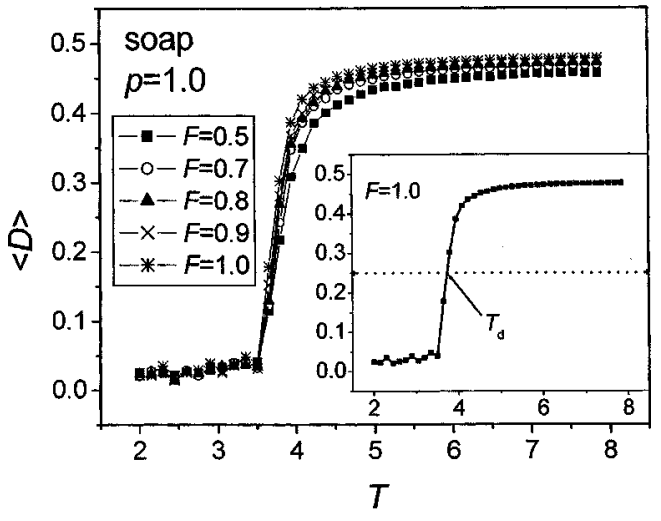

FIG. 2. The averaged damage for the soap structures with various size dimensions as a function of $T$ (in unit of $J / k_{\mathrm{B}}$ ). $F$ is the ratio of stopping radius of the damage spreading simulation over the maximum radius of the sample.

is the number of hexagons along the edge of the rhomb for which star-triangle transformation is applied.) Figure 2 and Fig. 4 are typical phase transition curves of the damage spreading of the soap structure and the 3-12 lattice with various $N$ or $F$. Generally, we define $T_{d}=\lim _{N \rightarrow \infty} T_{d}(N)$. However, as seen in Fig. 2 and Fig. 4, when $N$ is sufficiently large, $T_{\mathrm{d}}(N)$ does converge. We can use the temperature for a large $N$ as the approximate $T_{\mathrm{d}}$. We also observe the transition from frozen to chaotic state through the average damage $\langle D\rangle$ in Fig. 2 and Fig. 4. The maximum of $\langle D\rangle$ in the long time limit are all 0.5 , meaning that eventually damage spreads to all spins.

We see the important qualitative difference of DS on two classes of trivalent structures, the soap (and Voronoi) and the hierarchical lattice in Fig. 1. For the hierarchical lattice, $T_{\mathrm{d}}$ decreases sharply as 1- $p$ increases ( $p$ decreases) while for the Voronoi and soap, $T_{\mathrm{d}}$ remains nearly unchanged except for very small $p$. The behavior of soap and Voronoi are similar to the pure hexagonal lattice since in these structures the most probable polygons are hexagons.

\section{EFFECTS OF TRIANGLES ON DAMAGE SPREADING}

For a better understanding of the results of Fig. 1, one should pay more attention to the topology of the structures.

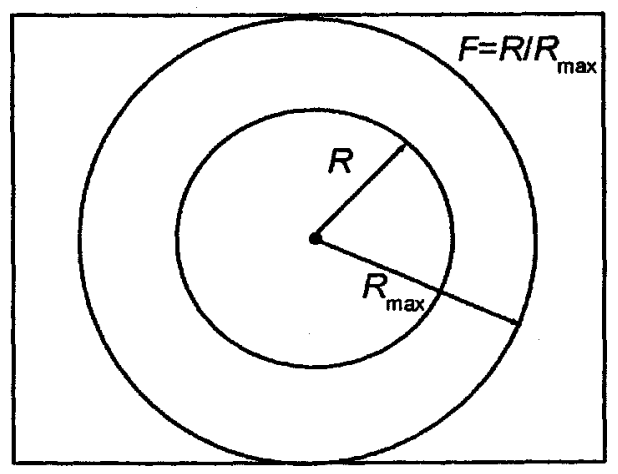

FIG. 3. Sketch of the size change of the soap and Voronoi structures. $F$ is the ratio of stopping radius of the damage spreading simulation over the maximum radius of the sample.

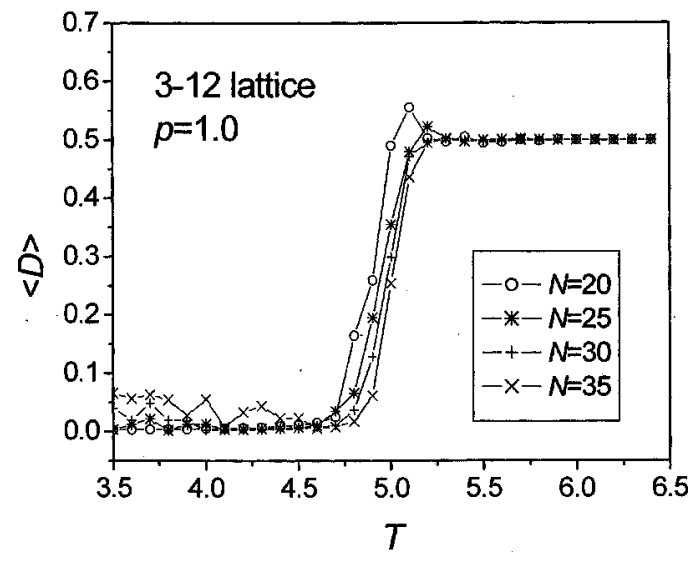

FIG. 4. The averaged damage for the 3-12 lattices with various size dimensions as a function of $T$ (in units of $J / k_{\mathrm{B}}$ ).

The transition from the frozen to the chaotic state in DS is influenced by two factors: temperature and the competition of the dynamics. In general Glauber dynamics favors a low energy state while Kawasaki dynamics favors a high energy state. When Glauber dynamics is dominant ( $p$ close to 1 ), the transition is determined mainly by temperature. In this case the transition temperature is higher. But as $p$ decreases, the contribution of Kawasaki dynamics plays a more important role, resulting in a lower transition temperature.

From Fig. 1, we observe that $T_{d}(6)<T_{d}(12)<T_{d}(24)$ $<T_{d}(48)$ for $p=1$, where $T_{d}(6), T_{d}(12), T_{d}(24)$, and $T_{d}(48)$ stand for the critical temperatures corresponding to the pure hexagonal, the 3-12, the 3-6-24, and the 3-6-12-48 lattice. This indicates that the more complicated the lattice, the larger the $T_{d}$ of the damage spreading becomes under Glauber dynamics. To understand this trend, the key point may be the numbers of triangles in the lattices. Note that the number of the triangles in the hexagonal, the 3-12, the 3-624, and the 3-6-12-48 lattices are respectively $0,2 N^{2}, 6 N^{2}$, and $18 N^{2}$, here $N$ is the size of the lattice (the number of the main polygons along one direction; see Ref. [5]). Although the number of the triangles are different among the 3-12, the 3-6-24, and the 3-6-12-48 lattices, the concentration of triangles in these lattices are the same (2/3) [5]. Since we have neglected the area influence on the DS in the calculation, we consider the triangle with the same level of importance as other polygons. Now the triangle has only three edges (the contiguity number $f=3$ ). When it is damaged, less cells will be affected, so the DS is difficult compared with the 6-gon $(f=6)$, the 12-gon $(f=12)$, the 24-gon $(f=24)$, and the 48gon $(f=48)$. Thus, for the lattices with larger number of triangles, bigger thermal noise is required to reach the equilibrium state (the so-called chaotic state), resulting in higher $T_{d}$. In pure Glauber dynamics, the damage is most readily healed in the triangle sites. However, in Kawasaki dynamics, damaged sites can be healed only in pairs while they diffuse and also create further damage. The Kawasaki dynamics involves two sites while the Glauber dynamics involves only single site.

First let us provide a qualitative picture of the competition between the Glauber and Kawasaki dynamics. From Fig. 1 we can see that the roles of the triangles on these two dy- 


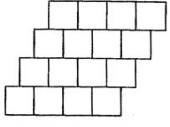

(a)

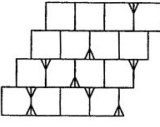

(b)

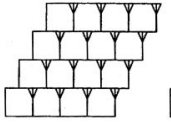

(c)

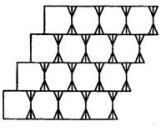

(d)
FIG. 5. (a) The topological hexagonal lattice. (b) The startriangle transformation $\left(N_{i}=10\right)$ made at the vertices randomly. (c) The 3-9 lattice. (d) The 3-12 lattice.

namics are very different. The role of the triangles varies with changing $p$ and the number of the triangles determines the trend of the $T_{\mathrm{d}}$ vs $(1-p)$ curves. From the inset of Fig. 1, we observe three zones for the 3-12, the 3-6-24, and the 3-6-12-48 lattices. In zone A, the triangles hinder the damage spreading, contrary to the case of zone $\mathrm{C}$, where the triangles enhance the damage spreading. Zone $\mathrm{B}$ is the transition region. It is the Kawasaki dynamics that causes the above change from zone A to $\mathrm{C}$.

Next we like to provide a more quantitative understanding of the effects of the number of the triangles on the trend of the $T_{\mathrm{d}}$ vs $(1-p)$ curves. We begin by designing an artificial trivalent cellular network where the number of the triangles can be changed gradually. Since only the topology of the structures is emphasized, we consider the simplest startriangle transformation in a hexagonal lattice. We do the transformation on the vertices of the topological hexagonal lattice with the dimension of $N \times N$ [Figs. 5(a) and 5(b)]. The transformation is made at the vertices at random. We notice that when the number $N_{i}$ of triangles inserted at vertices increases, the trend of the $T_{\mathrm{d}}$ vs $(1-p)$ curves does change. When the number of triangles approaches that of the 3-9 lattice [Fig. 5(c)], $T_{\mathrm{d}}$ decreases linearly with 1- $p$. When the number of triangles is further increased, we see similar trend of $T_{\mathrm{d}}$ as in the 3-12 lattice [Fig. 5(d)]. These are clearly shown in Fig. 6. Note that $N_{\mathrm{i}}$ is an average number over 100 configurations. For each configuration, the actual number of triangles inserted can be different from $N_{i}$, as the randomly generated points may coincide. For example, we may get only 9 triangles when we want to generate 10 triangles [see Fig. 5(b)].

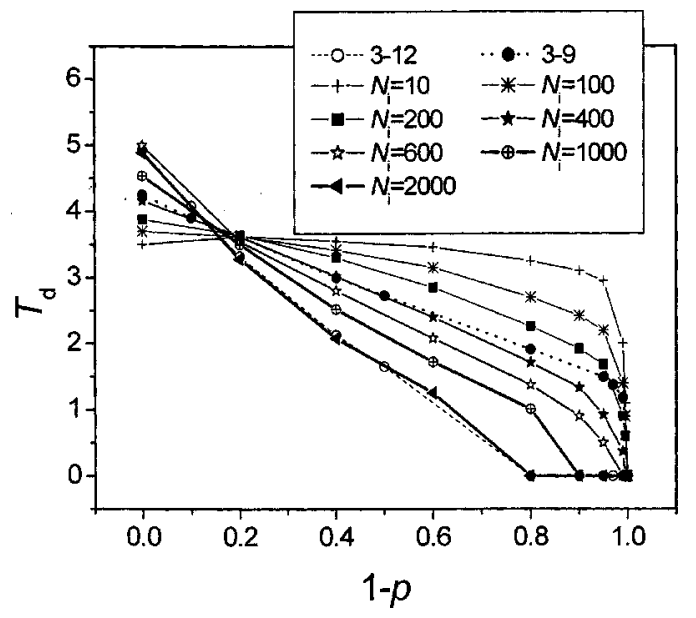

FIG. 6. The effect of the numbers of the triangles on the trend of the $T_{\mathrm{d}}$ vs $(1-p)$ curves $(N=20)$.

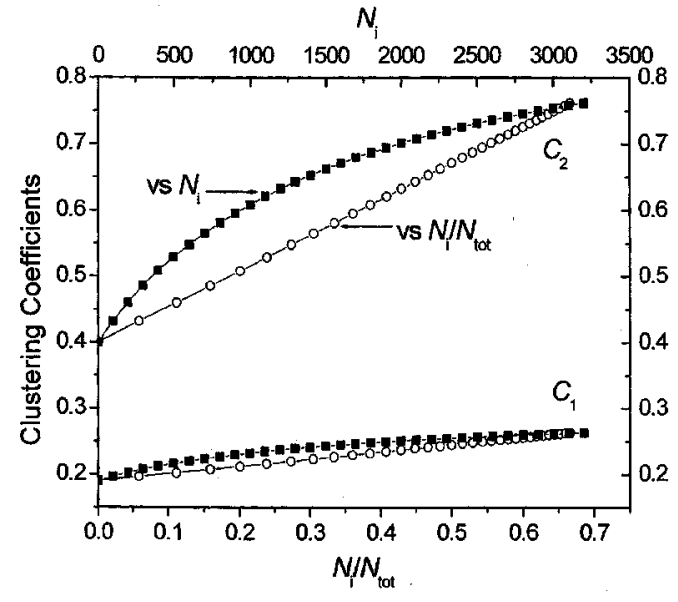

FIG. 7. The clustering coefficients of the network of Fig. 5(b) $(N=40)$ versus the number of inserted triangles (square) and the ratio of the inserted triangles to the total bubbles (hollow circle).

What is the key topological difference among the structures obtained by the insertion of triangles in the hexagonal network shown in Fig. 5, in relation to the trend of the damage temperature $T_{\mathrm{d}}$ shown in Fig. 6? We expect that clustering is an important topological property that greatly affects the evolution of damage spreading in the network. According to Newman [19], two measures can be defined to describe the clustering of a network (called the clustering coefficients):

$$
C_{1}=\frac{3 \times(\text { number of triangles })}{\text { (number of connected triples })}
$$

and

$$
C_{2}=\frac{1}{n} \sum_{i=1}^{n} c_{i}
$$

where $n$ is the number of nodes and $c_{i}$ is the ratio of the number of triangles connected to vertex $i$ over the number of triples centered on vertex $i$. Often $C_{2}$ is referred to as the average "network density" [19] and it is the average of the ratio, while $C_{1}$ is the ratio of the average. In the following discussion on the relation between clustering effect and damage spreading, we focus on $C_{2}$ since the dynamics of damage spreading is affected by the local clustering, and the proper average should be performed on each given vertex $i$. In any case, we have calculated the clustering coefficients of the networks in the sequence of structures in Fig. 5 and the results are shown in Fig. 7.

We also calculated these two values for Voronoi and soap froth structures studied here for comparison, which are respectively $C_{1}=0.293, C_{2}=0.425$ for Voronoi and $C_{1}=0.296$, $C_{2}=0.427$ for soap froth structure. Since our spins are put at the centers of the bubbles, the networks of spins are formed actually by connecting the centers of the bubbles (the dual lattice). When there is no inserted triangle, the hexagonal lattice has similar clustering property to Voronoi and soap structures. As we increase the number of the inserted triangles at the vertices of the hexagons, the average network 


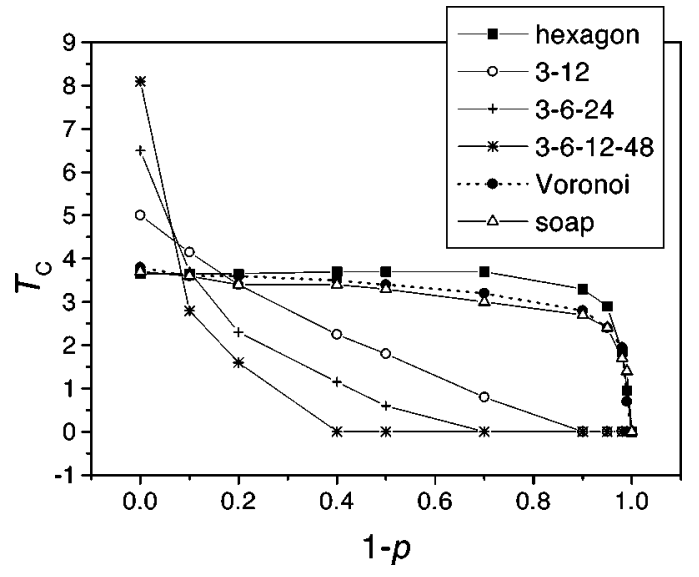

FIG. 8. The Curie temperatures of various structures versus $1-p$.

density $C_{2}$ is also increased. The increase in network density $C_{2}$ implies the increased effect of pairwise damage spreading via the neighbors of a given vertex $i$ through Kawasaki dynamics. As shown in Fig. 5, iteration of the star-triangle transformation results in more triangles inserted, leading to the increase of network density. For example, after the firstorder star-triangle transformation, one bubble turns into 3 bubbles (one hexagon into one 12-gon and two triangles). An increase of network density locally is equivalent to an increase of spin density, resulting in higher probability for spins to exchange with neighboring ones. In summary, increased number of inserted triangles leads to increased network density, which enhances the Kawasaki process. This is similar to the case of Potts model with the Swendson-Wang dynamics in which larger $q$ has lower transition temperature [20]. From this simple topological investigation, we obtain a heuristic understanding for the comparatively rapid drop in DS transition temperature for the hierarchical lattice as the weight of Kawasaki dynamics on DS is increased (1- $p$ increases) due to increased network density, as compared to the rather stable DS transition temperature in the soap and Voronoi cases.

Finally, when Kawasaki is dominant, damage always spreads, resulting in no transition. This agrees with the observations stated in Refs. [21,22], when Kawasaki dynamics is dominant in an Ising model. Thus we define $T_{\mathrm{d}}=0$ in the case of pure Kawasaki dynamics. This agrees with our simulation result in Fig. 1, where $T_{\mathrm{d}}$ of all of the structures approaches zero when 1-p approaches 1 .

\section{RELATION BETWEEN $T_{\mathrm{d}}$ AND $T_{C}$}

We depict the Curie temperatures of the above structures versus $1-p$ in Fig. 8 in order to compare $T_{\mathrm{d}}$ and $T_{C}$. The Curie temperatures are calculated using the crossover of the Binder's cumulants (see Fig. 9). For example, we get $T_{C}$ $=2.21 \pm 0.01$ for the 3-12 lattice with this method. From Fig. 8 we see that the transition temperature decreases, as the

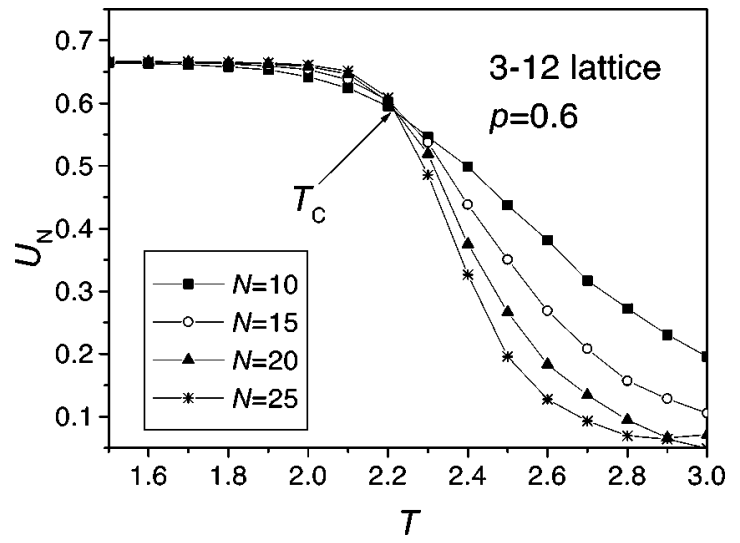

FIG. 9. Binder's cumulants as the function of temperature for the 3-12 lattice.

probability of the occurrence of the Kawasaki process increases. This is in agreement with previous works [23]. By comparing Fig. 1 with Fig. 8, we can say that $T_{\mathrm{d}}$ and $T_{C}$ in our two-dimensional trivalent structures are nearly the same. Since $T_{\mathrm{d}}$ varies with definitions (we can define the following temperatures as $T_{\mathrm{d}}$ : the temperature at $\langle D\rangle=\frac{1}{2}\langle D\rangle_{\max }[5]$, $\langle D\rangle=0$ [3], etc.), it is difficult to compare $T_{\mathrm{d}}$ with $T_{C}$ quantitatively. Thus we only want to comment that qualitatively these two temperatures are similar under competing GK dynamics.

\section{CONCLUSION}

In summary, we have studied the damage spreading of the Ising model on the two-dimensional trivalent structures with competing Glauber and Kawasaki dynamics and compared the DS transition temperature $T_{\mathrm{d}}$ and the Curie temperature $T_{C}$. We find that the two classes of trivalent structures exhibit different properties. The DS temperature $T_{\mathrm{d}}$ of the hierarchical lattices decreases sharply as 1- $p$ increases ( $p$ decreases), whereas for soap and Voronoi, $T_{\mathrm{d}}$ remains nearly unchanged till 1- $p$ is close to one. When Kawasaki dynamics is dominant ( $p$ is small) $T_{\mathrm{d}}$ and $T_{C}$ of all the structures approaches zero and no transition occurs. Finally, we see that $T_{\mathrm{d}}$ and $T_{C}$ in our two-dimensional structures exhibit similar behavior when we vary the weight $p$ of the competing GK dynamics. By inserting triangles on vertices of the hexagonal lattice, we also obtain a heuristic understanding for the difference between the DS behaviors in the two classes of structures. The rapid decrease of $T_{\mathrm{d}}$ for the hierarchical structure as the weight for Kawasaki dynamics increases is due to the increasing network densities of these structures, compared to the soap and Voronoi structures.

\section{ACKNOWLEDGMENT}

K.Y.S. acknowledges the support of Grant CERG 6071/ 02P. 
[1] H. Eugene Stanley, Dietrich Stauffer, Janos Kertesz, and Hans J. Herrmann, Phys. Rev. Lett. 59, 2326 (1987).

[2] F. G. Brady Moreira, Adauto J. F. de Souza, and Ananias M. Mariz, Phys. Rev. E 53, 332 (1996).

[3] F. D. Nobre, A. M. Mariz, and E. S. Sousa, Phys. Rev. Lett. 69, 13 (1992).

[4] Uriel M. S. Costa, J. Phys. A 20, L583 (1987).

[5] Z. Z. Guo, K. Y. Szeto, and Xiujun Fu, Phys. Rev. E 70, 016105 (2004).

[6] Pontus Svenson and Desmond A. Johnston, Phys. Rev. E 65, 036105 (2002).

[7] K. Y. Szeto, Xiujun Fu, and W. Y. Tam, Phys. Rev. Lett. 88, 138302 (2002).

[8] K. Y. Szeto and W. Y. Tam, Phys. Rev. E 53, 4213 (1996).

[9] R. Liebmann, in Statistical Mechanics of Periodic Frustrated Ising Systems, edited by H. Araki et al., Lecture Notes in Physics (Springer-Verlag, Berlin, 1986).

[10] K. Y. Lin and C. C. Chang, Int. J. Mod. Phys. B 16, 1241 (2002).

[11] J. Oitmaa and M. Keppert, J. Phys. A 35, L219 (2002).
[12] S. Bekhechi, A. Benyoussef, B. Ettaki, M. Loulidi, and A. El Kenz, Phys. Rev. E 64, 016134 (2001).

[13] Attila Szolnoki, Phys. Rev. E 62, 7466 (2000).

[14] Ce-Jun Liu, H.-B. Schuttler, and Jia-Zhen Hu, Phys. Rev. E 65, 016114 (2001).

[15] Peter Grassberger, J. Phys. A 28, L67 (1995).

[16] M. M. Xavier, Jr., F. D. Nobre, A. M. Mariz, and F. A. da Costa, Phys. Lett. A 267, 127 (2000).

[17] M. Leticia Rubio Puzzo and Ezequiel V. Albano, Physica A 293, 517 (2001).

[18] H. Hong, Beom Jun Kim, and M. Y. Choi, Phys. Rev. E 66, 018101 (2002).

[19] M. E. J. Newman, SIAM Rev. 45, 167 (2003).

[20] E. M. de Sousa Luz, M. P. Almeida, U. M. S. Costa, and M. L. Lyra, Physica A 282, 176 (2000).

[21] T. Vojta, cond-mat/9803053.

[22] Sharon C. Glotzer and Naeen Fan, Physica A 173, 325 (1991).

[23] J. M. Gonzalez-Miranda, P. L. Garriodo, J. Marro, and J. L. Lebowitz, Phys. Rev. Lett. 59, 1934 (1987). 